\title{
INCORPORAÇÃO DE RESÍDUOS DE PEDRA ORNAMENTAL NO DESENVOLVIMENTO DE CERÂMICA ARGILOSA PARA APLICAÇÃO DE PAVIMENTOS*
}

Artur Camposo Pereira ${ }^{1}$ Sergio Neves Monteiro ${ }^{2}$ Foluke Salgado de Assis ${ }^{3}$ Fabio Oliveira Braga ${ }^{4,5}$

\section{Resumo}

Cerâmicas argilosas foram processadas com adição de até $30 \%$ em peso de resíduos de pedra ornamental e queimadas a $900,950,1000$ e $1050^{\circ} \mathrm{C}$. Esta adição contribuiu para ajustar a plasticidade do corpo da argila e aumentar a densidade seca aparente, que ocasiona um empacotamento mais elevado das partículas. A cerâmica adicionada diminuiu sua retração linear com a quantidade de resíduos e evita assim alterações dimensionais excessivas nos produtos finais. Outra vantagem foi a diminuição na absorção de água com resíduos adicionados. As resistências à flexão e à compressão sofreram uma diminuição associada à adição de resíduos. Isto foi atribuído à grande quantidade de quartzo nos resíduos, que gera rachaduras e enfraquece o produto final. No entanto, até $30 \%$ em peso de adição a resistência mecânica, de acordo com a norma ASTM, é ainda satisfatória para utilização como cerâmica de pavimentação.

Palavras-chave: Pavimento Cerâmico, Resíduos, Rocha Ornamentais, Argila Amarela.

\section{DEVELOPMENT OF ORNAMENTAL STONE WASTE ADDED CLAY CERAMIC FOR PAVEMENT APPLICATION}

\begin{abstract}
Clay ceramic were processed with addition of up to $30 \mathrm{wt} . \%$ of ornamental stone waste and fired at $900,950,1000$ and $1050^{\circ} \mathrm{C}$. This addition contributed to adjust the clay body plasticity and increaser the dry bulk density, which indirect a higher packing of particles. The added ceramic decreased its linear shrinkage with the amount of waste and so avoid excessive dimensional changes in the final products. Another advantage was the decrease in water absorption with added waste. Both the flexural and compressive strengths suffered a decrease associated with the addition of waste. This was attributed to the large amount of quartz in the waste, which generates cracks and weakens the final product. However, up to $30 \mathrm{wt} . \%$ addition the mechanical strength, according to the ASTM standard, is still satisfactory for use as ceramic paver.
\end{abstract}

Keywords: Ceramic Paver, Waste, Ornamental Rock, Yellow Clay.

1 Instituto Militar de Engenharia - IME, Seção de Engenharia Mecânica e de Materiais, Praça General Tibúrcio, 80, Rio de Janeiro, 22290-270, RJ, Brasil.

2 Instituto Militar de Engenharia - IME, Seção de Engenharia Mecânica e de Materiais, Praça General Tibúrcio, 80, Rio de Janeiro, 22290-270, RJ, Brasil.

3 Instituto Militar de Engenharia - IME, Seção de Engenharia Mecânica e de Materiais, Praça General Tibúrcio, 80, Rio de Janeiro, 22290-270, RJ, Brasil.

4 Instituto Militar de Engenharia - IME, Seção de Engenharia Mecânica e de Materiais, Praça General Tibúrcio, 80, Rio de Janeiro, 22290-270, RJ, Brasil.

5 Faculdade SENAI Rio, Rua Mariz e Barros, 678, 20270-003, Rio de Janeiro, RJ, Brasil.. 


\section{INTRODUÇÃO}

A cerâmica argilosa é usada mundialmente na construção civil como os produtos distintos tais como tijolos, blocos, telhas e tubulação. Cada um desses produtos para uma determinada aplicação requer propriedades técnicas especificadas por padrões. Por exemplo, uma cerâmica argilosa de pavimentação é um produto utilizado para cobrir ruas, calçadas e pavimentos de pavimentos ilustrados na Figura 1.

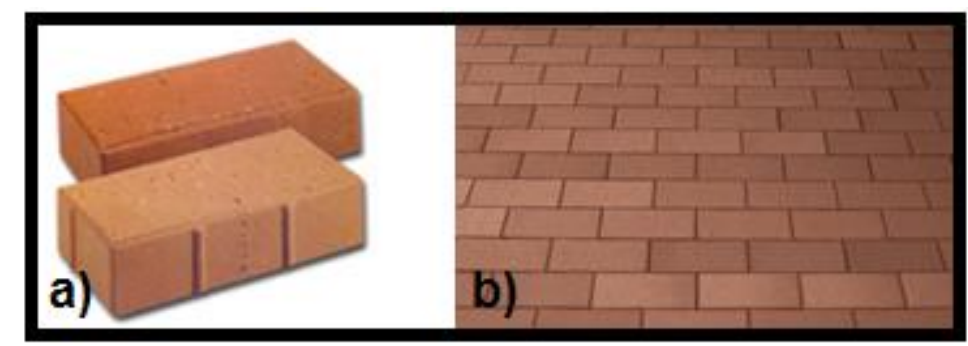

Figura 1. a) Cerâmica argilosa de pavimentação e b) Pavimentos cerâmicos aplicados sobre um piso de pavimento.

A cerâmica de pavimentação na Figura 1 proporcionam benefícios a um pavimento, tais como longa vida, alta resistência mecânica, cores naturais exuberantes, bem como facilidade de implantação e reparação. Para atingir essas características, tanto a argila como seu processamento devem atender às especificações técnicas estabelecidas pelas normas. Neste trabalho foi estudado o desenvolvimento de pavimentos fabricados com argila do município de Campos dos Goytacazes, no norte do estado do Rio de Janeiro, para reduzir custos e solucionar um problema ambiental regional de acumulação de resíduos de pedras ornamentais. Para o Brasil, essa é uma questão relevante, já que a indústria de cerâmica argilosa representa cerca de $5 \%$ da produção industrial do país [1] e quantidade notável é exportada [2]. No entanto, quanto aos pavimentos cerâmicos de cerâmica, não há padrões brasileiros relacionados. Portanto, 0 presente trabalho considerou as normas ASTM $[3,4]$ para avaliação de propriedades.

Além da cerâmica de argila, o Brasil tem também uma importante produção de pedras ornamentais sendo um dos maiores exportadores mundiais [5]. As indústrias localizadas no norte do Estado do Rio de Janeiro geram uma quantidade considerável de resíduos de pedras ornamentais. Isso é indevidamente descartado, causando danos ao meio ambiente e à saúde humana [6,7]. Esta situação motivou o desenvolvimento e a caracterização de cerâmica argilosa de pavimentação adicionada com resíduos de pedras ornamentais.

\section{MATERIAIS E MÉTODOS}

O resíduo de rocha ornamental e a argila foram secos durante 24 horas em um forno de marca Odontobrás, modelo EL-1.5, a uma temperatura de $110^{\circ} \mathrm{C}$. Após secagem, as matérias primas foram esmagadas manualmente e peneiradas através de malha 42.

Prepararam-se formulações com adições de 0, 10, 20 e 30\% em peso de resíduos. A composição de $0 \%$ serve de referência. As misturas foram homogeneizadas num misturador seco durante 15 minutos, humedecidas com $8 \%$ em peso de água e peneiradas em malha 20. 
Foram feitas amostras de base retangular com dimensões de $115 \mathrm{~mm}$ x $25 \mathrm{~mm}$ com um peso de $60 \mathrm{~g}$ da mistura em equilíbrio JH2102, marca Bioprecisa e pressão uniaxial a $34 \mathrm{MPa}$.

Após a preparação, as amostras foram colocadas num forno a $110^{\circ} \mathrm{C}$, onde foi secado durante 24 horas. Após secagem, as amostras foram pesadas, medidas e aquecidas num forno modelo FL 1300 MAITEC, a 900, 950, 1000 ou $1050^{\circ} \mathrm{C}$, a uma taxa de aquecimento constante de $2^{\circ} \mathrm{C} / \mathrm{min}$. $\mathrm{O}$ nível de temperatura foi mantido durante 3 horas e, em seguida, as amostras foram esfriadas em temperatura ambiente. Após a queima e resfriamento, as amostras foram novamente pesadas e medidas com um paquímetro digital Mitutoyo e foram determinadas as seguintes propriedades físicas e mecânicas: densidade seca aparente, retração linear, absorção de água, resistência à ruptura por flexão e resistência à compressão. A densidade aparente foi medida dividindo a massa seca pelo volume. A retração linear foi determinado pelo comprimento das amostras antes e depois da queima. A absorção de água foi obtida de acordo com o procedimento padrão [8]. A resistência à ruptura por flexão [9] e a resistência à compressão foram determinadas numa máquina de ensaio universal Instron 5582.

Novas amostras com dimensões de $25 \mathrm{~mm} \times 25 \mathrm{~mm}$ foram cortadas a partir das amostras retangulares, utilizando um cortador mecânico MINITON. Estes foram utilizados para determinar a resistência à compressão.

A distribuição de tamanho de partícula da argila foi realizada de acordo com o padrão NBR 7181-84 (ABNT), que combina técnicas de peneiramento e sedimentação [10]. A plasticidade das argilas foi determinada pelo método de Atterberg, de acordo com as normas técnicas [11,12].

O ensaio dilatométrico foi realizado no equipamento Netzsch DIL 402 PC, com uma taxa de aquecimento de $10^{\circ} \mathrm{C} / \mathrm{min}$ e uma temperatura final de $1050^{\circ} \mathrm{C}$. Para $\mathrm{O}$ ensaio, foram feitas duas amostras de $2 \mathrm{~g}$, uma sem resíduos e a outra com $30 \%$ em peso de resíduos, em formato cilíndrico pressionado com 1 tonelada.

Para se obterem resultados de difração de raios $X$ de 0 e $30 \%$ do resíduo a todas as temperaturas, utilizou-se o difratômetro XRD7000 da marca Shimadzu e operou-se com radiação $\mathrm{Cu}$-Ka para uma gama de $2 \theta$ de $5^{\circ}$ a $60^{\circ}$, com passo de $0,02^{\circ}$ e tempo de 5 s.

\section{RESULTADOS E DISCUSSÃO}

Na Figura 2, são apresentados os padrões de raios $X$ de queima das massas com 0 e $30 \%$ de resíduo, a temperaturas de $900,950,1000$ e $1050^{\circ} \mathrm{C}$. Pode-se observar que o quartzo está muito presente em todas as massas e em todas as temperaturas. E considerado residual, originando-se tanto da argila e do resíduo, e atua como um redutor de plasticidade. No entanto, em quantidade excessiva, isto pode causar uma diminuição na resistência mecânica, devido às fissuras criadas no seu processo de transformação alotrópica a $573^{\circ} \mathrm{C}$. Deve-se notar a presença de mica muscovita, que não é apenas mostrada a $1050^{\circ} \mathrm{C}$ e a $1000^{\circ} \mathrm{C}$, sem resíduos. $\mathrm{A} 950^{\circ} \mathrm{C}$, sem a adição do resíduo, o rutilo aparece em pequenas quantidades. No entanto, com a adição de resíduo, cordierite que é encontrado em pequenas quantidades. A mullita mostrada a $1050^{\circ} \mathrm{C}$ foi formada pela decomposição de metacaolinita. Pode-se notar a presença de feldspatos, como um albito, anorthite e orthoclase, fluxos importantes, que favorecem a sinterização e diminuem a porosidade. Observa-se que a adição do resíduo provocou o aumento dos componentes formadores de fase líquida. 

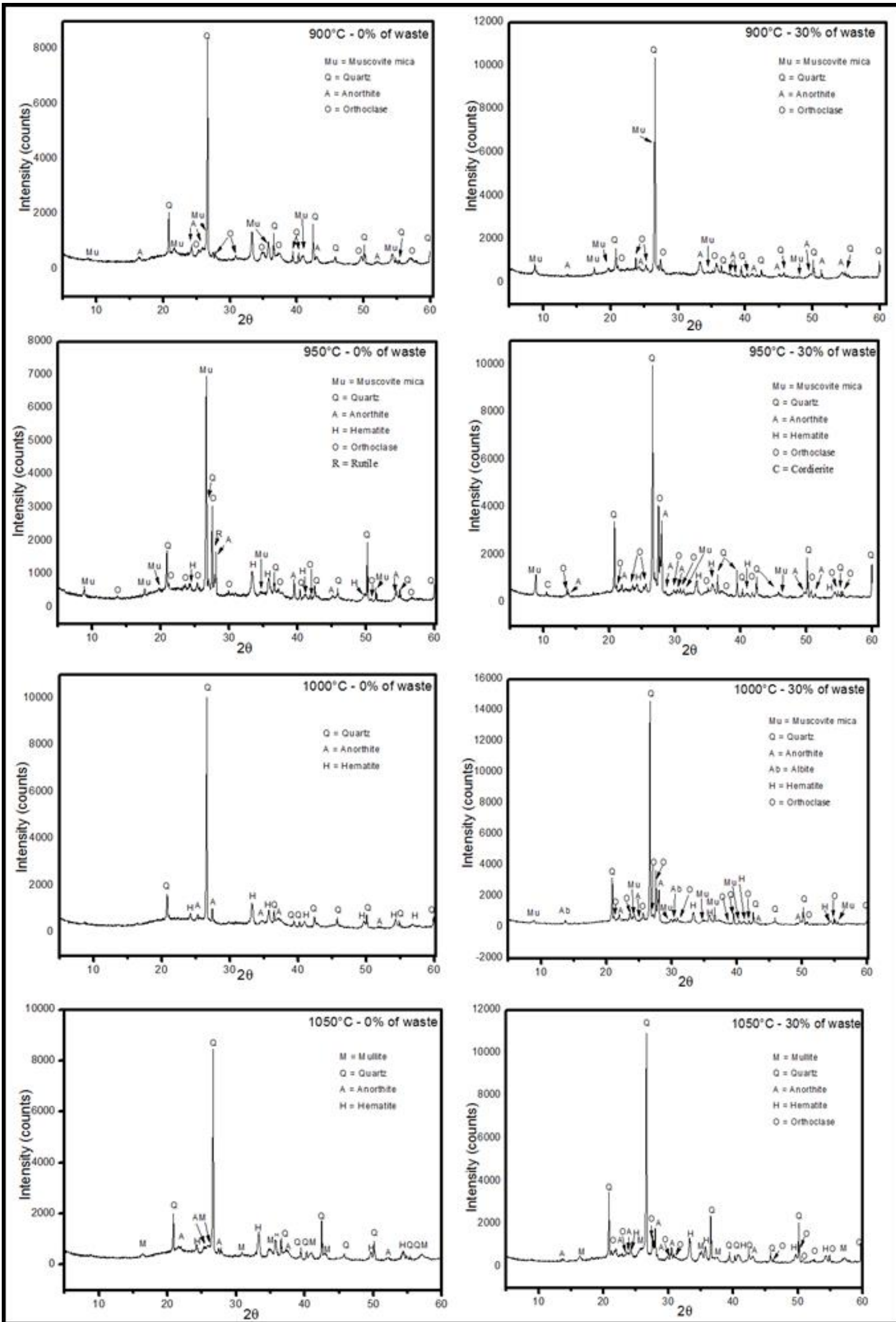

Figura 2. Padrões de difração de raios $X$. 
A Figura 3 representam o comportamento das curvas de dilatometria com 0 e $30 \%$ de resíduo respectivamente. Entre 100 e $200^{\circ} \mathrm{C}$ as curvas apresentam uma inclinação, representando uma contração devido à eliminação da humidade da água. A partir de $200^{\circ} \mathrm{C}$ as curvas estabilizam a $500^{\circ} \mathrm{C}$, quando sofrem uma queda súbita devido à transformação da caulinita em metacaulinita. Observa-se uma alteração na inclinação das curvas, ocorrendo em valores mais elevados para a formulação com $0 \%$ de resíduo (cerca de $650^{\circ} \mathrm{C}$ ). O processo de sinterização se destaca, mas a transformação de quartzo é capaz de retardar a contração, que está presente na massa com $30 \%$ do resíduo.

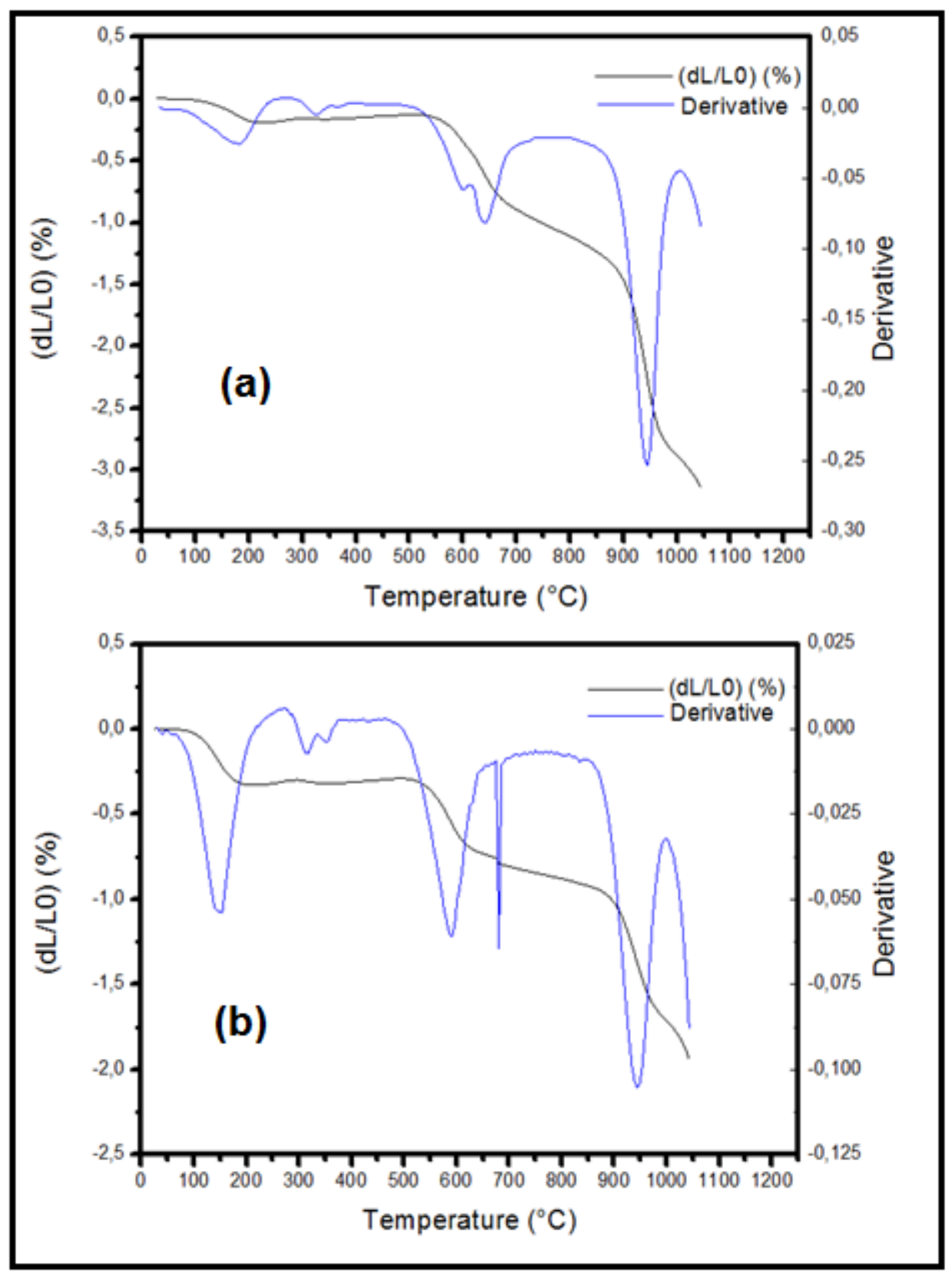

Figura 3. Dilatometria da formulação com (a) $0 \%$ e (b) $30 \%$ de resíduo.

A Figura 4 (a) mostra a distribuição do tamanho das partículas da argila e do resíduo. A fração abaixo de $2 \mu \mathrm{m}$ representa a fração de argila, entre $2 \mu \mathrm{m}$ e $20 \mu \mathrm{m}$ a fração de silte e acima de $20 \mu \mathrm{m}$ de fração de areia. Apesar do resíduo ter uma 
granulometria mais grosseira do que a argila, tem granulometria fina suficiente para ser utilizada na formulação das massas. O resíduo de rocha ornamental é um material não plástico, o que pode melhorar o empacotamento das amostras e diminuir o encolhimento linear.

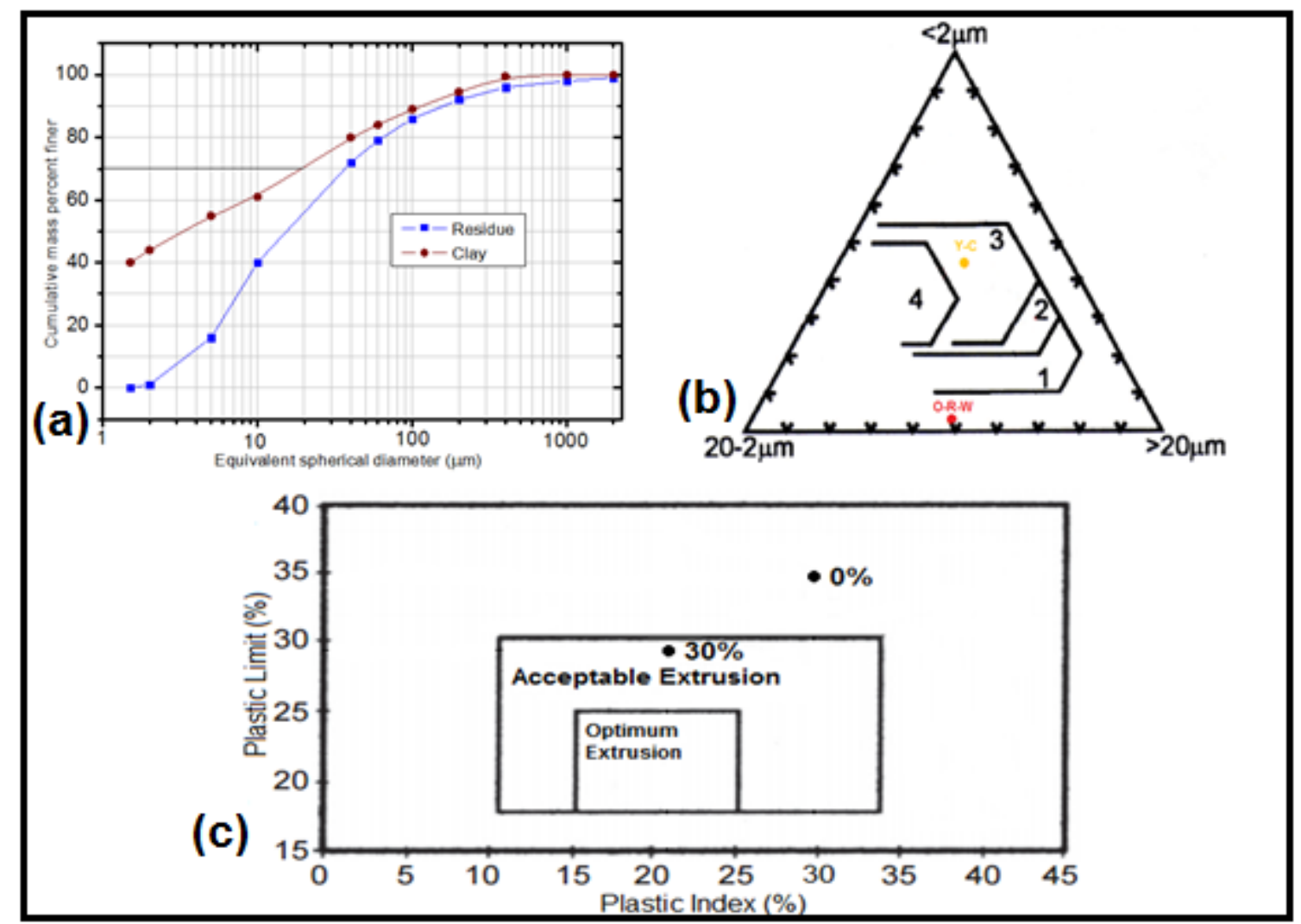

Figura 4. (A) Distribuição do tamanho das partículas das matérias-primas. (B) Diagrama de Winkler com locais de resíduos amarelos $(\mathrm{Y}-\mathrm{C})$ e rochas ornamentais (O-R-W). (C) Prognóstico da extrusão das massas com 0 e $30 \%$ de resíduo.

A Figura 4 (b) apresenta a localização das argilas dentro do Diagrama de Winkler. Este diagrama baseia-se na granulometria de matérias-primas para delinear regiões adequadas para o fabricação de determinados produtos tais como tijolo maciço (região 1), tijolo oco (região 2), ladrilhos (região 3) e grilhões (região 4).

A Figura 4 (c) apresenta um prognóstico da extrusão das massas com 0 e $30 \%$ de resíduo por meio dos limites de Atterberg [13]. Para isso, um limite de plástico, que indica a quantidade mínima de água para atingir o estado plástico e limite de líquido, que indica a quantidade máxima de água que o material sem alterar seu estado plástico. Considera-se na literatura que a massa de argila ou argila tem um índice plástico mínimo de 10\% [14].

$\mathrm{Na}$ Figura 5 (a), pode-se observar que os valores das massas com 0 e $10 \%$ de incorporações de resíduos são estatisticamente semelhantes. Com a adição de $20 \%$ a densidade seca apresenta um aumento, sendo semelhante a $30 \%$. O resíduo de rocha ornamental apresentando partículas grosseiras, quando comparado com a argila, causando um aumento no empacotamento das partículas e aumentando a densidade, explica este aumento, com maior conteúdo de incorporação. Isso também acontece porque as massas com menor teor de resíduos têm uma quantidade maior de argila. Estes apresentam uma pequena diferença na partícula tamanho, o que provoca menos embalagem [15]. 

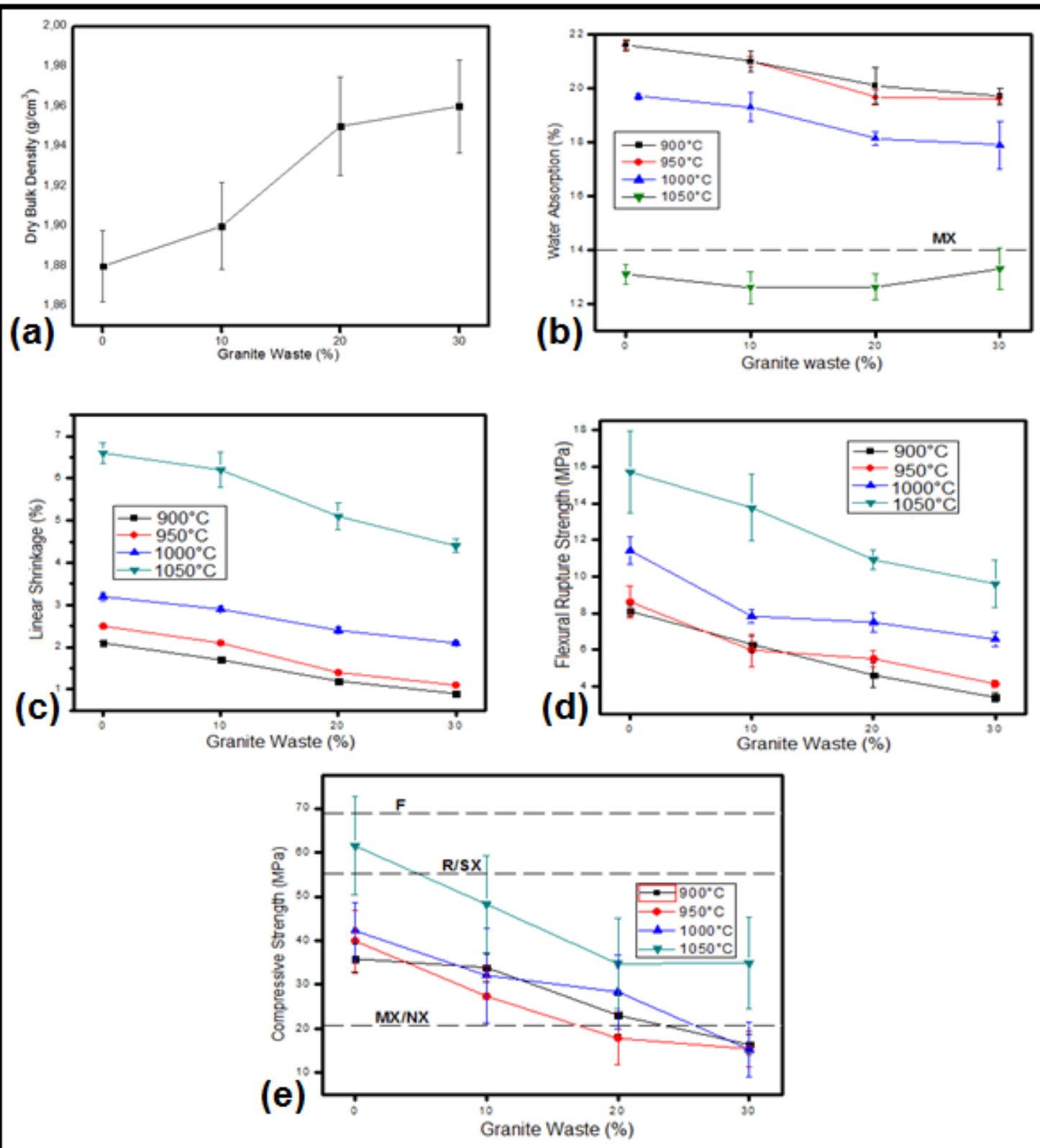

Figura 5. Influência do teor de resíduos de rochas ornamentais em espécimes queimadas a 900, 950, 1000 e $1050^{\circ} \mathrm{C}$ : a) densidade seca, b) absorção de água, c) retração linear, d) resistência à flexão, e) resistência à compressão.

A Figura 5 (b) mostra os resultados de absorção de água para as amostras incorporadas com resíduos a todas as temperaturas de queima. Note-se que, a temperaturas mais elevadas, a absorção de água diminui. Este fato é explicado pela sinterização mais eficiente a temperaturas mais elevadas, resultando em um maior fechamento dos poros abertos, reduzindo assim a absorção de água.

A retração linear, mostrado na Figura 5 (c), aumenta com o incremento de temperatura. Isto é justificado por sinterização mais eficiente a temperaturas mais elevadas que resulta num maior fechamento de poros e as peças têm uma redução nas suas dimensões. 
As Figuras 5 (d) e 5 (e) mostram os resultados da resistência à ruptura por flexão e resistência à compressão, respectivamente. A queima a altas temperaturas, geralmente, beneficia a resistência mecânica, devido a uma melhor sinterização das partículas, resultando numa melhoria na consolidação. Isso pode estar ocorrendo porque o resíduo de rocha ornamental tem uma grande quantidade de quartzo. Ela sofre transformação alotrópica a $573^{\circ} \mathrm{C}$, gerando tensões dentro das peças e resultando em micro rachaduras que atuam como concentradores de tensão contribuindo para a redução da resistência mecânica [15].

A Figura 6 mostra as micrografias ópticas de espécimes sem resíduo e com 30\% em peso de resíduo. É possível notar que há um aumento na quantidade de partículas de quartzo e seu tamanho. 

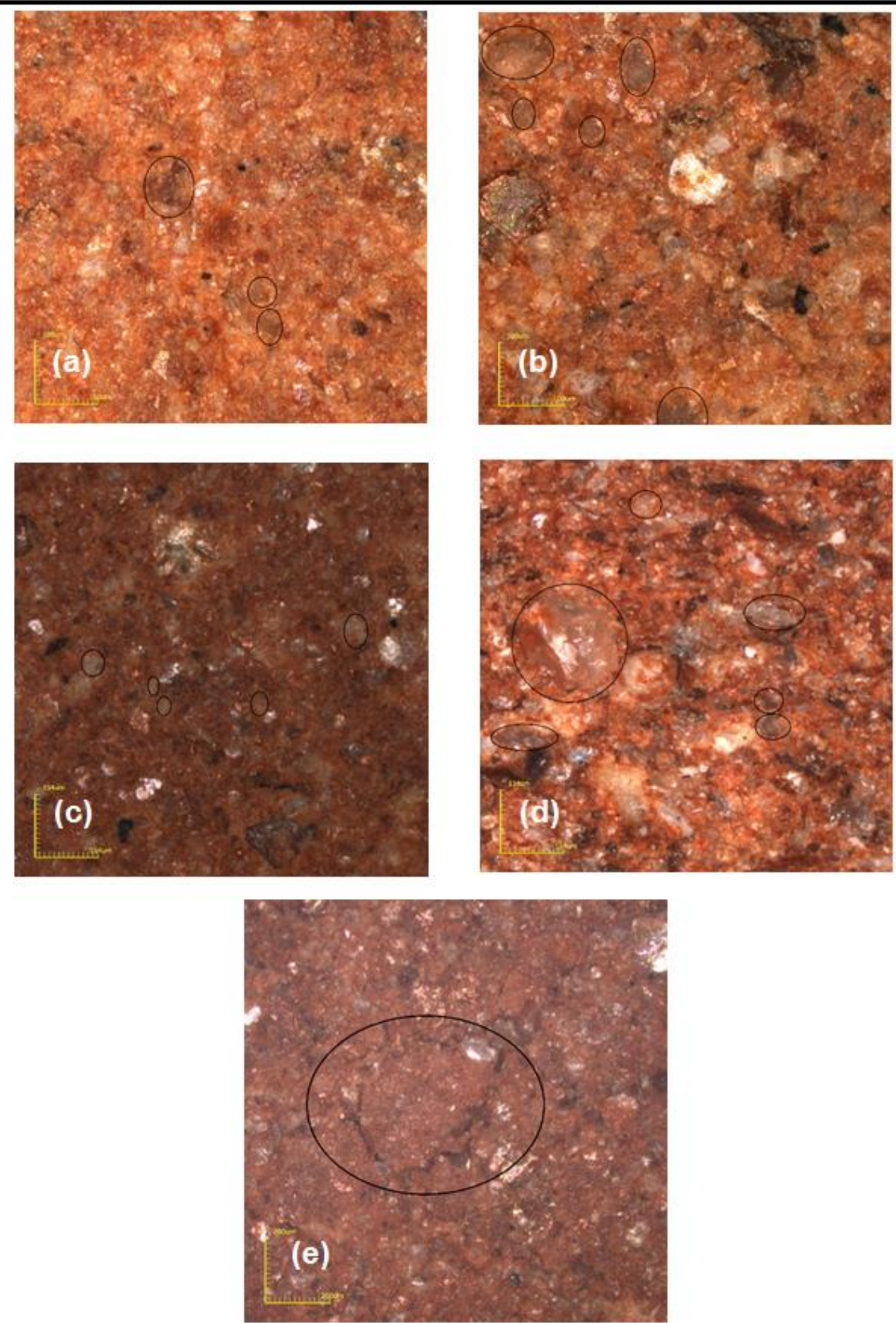

Figura 6. Micrografias ópticas de amostras de ensaio: a) Queima a $950^{\circ} \mathrm{C}$, sem resíduos, com aumento de $430 x$; B) Queima a $950^{\circ} \mathrm{C}$, com $30 \%$ em peso de resíduos, com aumento de 430x; C) Queima a $1050^{\circ} \mathrm{C}$, sem resíduos, com aumento de $216 x$; D) Despedido a $1050^{\circ} \mathrm{C}$, com $30 \%$ em peso de resíduos, com aumento de $216 \mathrm{x}$. E) com $30 \%$ em peso de resíduo disparado a $1050^{\circ} \mathrm{C}$, aumento de $216 x$. 
Apesar da redução da resistência à compressão, que atinge $42 \%$ a $1050^{\circ} \mathrm{C}$ com $30 \%$ em peso de resíduos, praticamente todas as formulações a todas as temperaturas de queima são satisfatórias, de acordo com a ASTM, para a produção de algum tipo de cerâmica de pavimentação.

\section{CONCLUSÃO}

- O resíduo ajustou a plasticidade da argila utilizada permitiu, por granulometria grosseira, um maior empacotamento, aumentando a densidade seca.

- As peças quando submetidas a temperaturas de queima mais altas apresentaram melhores resultados na sinterização, apresentando melhora em todas as propriedades investigadas.

- O aumento do teor de resíduos resultou em uma menor retração linear, o que é de extrema importância para o dimensionamento das peças.

- Na absorção de água, o resíduo provocou pequenas alterações, diminuindo-as.

- Devido às prováveis fissuras geradas pela grande quantidade de quartzo presente no resíduo, houve uma redução significativa na resistência mecânica com o aumento do teor residual.

- O uso de resíduos de rochas ornamentais é viável para alguns tipos de cerâmicas de pavimentação. Devido aos resultados de absorção de água e resistência à compressão obtidos, podem ser produzidos pavimentos do tipo MX e NX com uma temperatura de queima de $1050^{\circ} \mathrm{C}$, de acordo com ASTM.

- Além das melhorias de algumas propriedades da cerâmica, a utilização deste resíduo para este fim permite a reciclagem de um material que poderia ser descartado indevidamente e causando danos ao meio ambiente.

\section{Agradecimentos}

Os autores agradecem às agências brasileiras CNPq e FAPERJ pelo apoio a essa investigação.

\section{REFERÊNCIAS}

1 G. M. Bustamante, J. C. Bressiani. Industrial Ceramics. 2016, 5, 3-5.

2 R. M. Pinheiro, V. S. Candido, S. N. Monteiro, C. M. F. Vieira, Cerâmica. 2013, 59, 350, 310-316.

3 ASTM - American Society for Testing and Materials. (2014) Standard Specification for Heavy Vehicular Paving Brick, C1272, 2014.

4 ASTM - American Society for Testing and Materials. (2014) Standard Specification for Pedestrian and Light Traffic Paving Brick, C902, 2014.

5 R. R. Menezes, H. S. Ferreira, G. A. Neves, H. L. Lira, H. C. Ferreira; J. Eur. Ceram. Soc. 2005, 25, 1149.

6 C. Simsek, Z. Karaca, U. Gemini, O. Gunduz; Fresenius Environ. Bull. 2005, 14, 1013.

7 G. Rego, C. Martinez, A. Quero, T. P. Blanco, J. M. Borquea; Clinical Medicine 2001, 116, 291.

8 ASTM - American Society for Testing and Materials. Water Absorption, Bulk Density, Apparent porosity, and Apparent Specific Gravity of Fired Whiteware Products C373 (1972).

9 ASTM - American Society for Testing and Materials. Flexural Properties of Ceramic Whiteware Materials C674 (1997).

10 NBR - 7171-84, Brazilian Association of Technical Standards - ABNT. Rio de Janeiro, 1984, Brazil. 
11 Brazilian Association of Technical Standards - ABNT, Determination of the Plasticity Limit, NBR - 7180, Rio de Janeiro,1984, Brazil.

12 Brazilian Association of Technical Standards ABNT, Determination of the Liquidity Limit, NBR - 6459, Rio de Janeiro,1984, Brazil.

13 M. Marsigli, M. Dondi, L'Industria dei Laterizi 46 (1997) 214-222.

14 M. F. Abajo, Manual sobre Fabricación de Baldosas, Tejas y Ladrillos, Beralmar S. A, Espanha (2000) 130.

15 J. P. V. T. Manhães, J. M. S. Moreira, J. N. F. Holanda. Cerâmica. 2009, 55. 371-378. 